\title{
Tangence
}

\section{L’esthétique hyperréaliste de Bret Easton Ellis}

\section{Frances Fortier}

Numéro 44, juin 1994

La référence littéraire

URI : https://id.erudit.org/iderudit/025816ar

DOI : https://doi.org/10.7202/025816ar

Aller au sommaire du numéro

Éditeur(s)

Tangence

ISSN

0226-9554 (imprimé)

1710-0305 (numérique)

Découvrir la revue

Citer cet article

Fortier, F. (1994). L'esthétique hyperréaliste de Bret Easton Ellis. Tangence, (44), 94-105. https://doi.org/10.7202/025816ar d'utilisation que vous pouvez consulter en ligne.

https://apropos.erudit.org/fr/usagers/politique-dutilisation/ 


\section{L'esthétique hyperréaliste de Bret Easton Ellis Frances Fortier}

Comment peut-on mentir ainsi? Comment peut-il nous raconter de telles conneries?

American psycho (509)

American psycho est le récit des meurtres commis par un serial killer de bonne famille, élégant à l'excès, pourri de fric et de coke, et dont les deux passions majeures consistent à bichonner son propre corps et à supplicier celui de ses victimes. Satire mordante des mœurs contemporaines, ce troisième ouvrage de Bret Easton Ellis met en scène la vulgarité de la société américaine, surtout lorsqu'elle se targue de raffinement. Le caractère subversif de l'ouvrage réside moins, cependant, dans l'acte de dénonciation que dans le registre utilisé: American psycho est lui-même vulgaire, puisant ironiquement aux formes littéraires déclassées, à mi-chemin entre le film d'horreur et la bande dessinée, sur fond de pornographie et de rock'n roll.

Texte-limite, American psycho déboute toutes les conceptions modernes de la littérature. Le procès de la signifiance s'inverse ici dans un véritable culte de la futilité, de l'in-signifiance, du nonmotivé; le portrait social, minutieusement tracé, est tellement outrancier qu'il se désigne comme artifice ; l'écriture, froide, descriptive, à l'image d'un viseur de caméra, est neutre au point de masquer tout travail du signe; enfin, le pilonnage systématique du code réaliste, lié à la sollicitation constante de formes culturelles mineures, dénie toute inscription dans la tradition littéraire et sanctionne la dimension postmoderne de cette écriture.

C'est par une exploitation subversive de la référence que s'accomplit, en ce texte, la "mort" de la littérature. L'omniprésence de la référence extra-textuelle, où la présence du réel est cultivée avec acharnement, se double du gommage intentionnel de la bibliothèque, remplacée par une esthétique du spectaculaire. Parallèlement, en une contestation de la modernité, aucune allusion métatextuelle ne vient signaler une quelconque apparte- 
nance à la sphère littéraire. Sciemment, le texte opère à la frontière de la réalité et de la fiction et joue allègrement en marge du canonique.

\section{L'effet réaliste objectif}

Répugnant. Horrifiant. Abject. Obscène. American psycho fait scandale et Bret Easton Ellis, dit-on, est pris à partie, vilipendé, injurié, menacé de mort. L'indignation se porte garante du succès de librairie: les ventes s'accumulent au gré des condamnations féministes et des protestations de l'opinion publique. Le roman, certes sulfureux, conjugue violence, pornographie et meurtres en série avec un détachement et une insensibilité saisissants. Tous les tabous contemporains sont pulvérisés - cruauté maniaque, mépris des femmes, des pauvres et des homosexuels, anthropophagie - en une froide distanciation de la rectitude politique.

Pour sa défense, on arguera du statut fictif de l'ouvrage. Le paratexte de la plus récente édition française multipliera les précautions: le quatrième de couverture précise le sens de l'ouvrage en termes de "réquisitoire lucide et froid d'une Amérique autosatisfaite" cependant qu'une "Note de l'auteur" spécifie, selon la formule consacrée, qu'il s'agit d'une ouvre de fiction. En outre, une présentation signée Michel Braudeau du Monde détaille les circonstances de production et de réception de l'ouvrage, reprend les arguments de Norman Mailer et d'Umberto Eco qui ont soutenu Ellis en démarquant la fiction de l'intention, et insiste sur les qualités stylistiques d'American psycho ${ }^{1}$.

La prégnance du statut référentiel est telle qu'elle obnubile à première vue le projet esthétique de l'ouvrage. De fait, American psycho, en n'affichant pas son appartenance au littéraire, s'ingénie à pervertir le lien entre la vérité et la fiction par la multiplication

1 Paru en 1993 dans la collection *Points roman * du Seuil après une première édition en 1992 chez Salvy, l'édition française d'American Psycbo, dans une traduction d'Alain Defossé, répète l'histoire éditoriale de la version originale. De fait, le manuscrit américain s'est vu refusé par la maison Simon \& Shuster, éditeur des précédents romans d'Ellis et qui lui avait précisément commandé cet ouvrage sur un serial killer new-yorkais. La maison Vintage prend le relais et publie, en 1991, la première édition américaine. Le même scénario se reproduit outre-Atlantique: Christian Bourgois, qui avait fait paraitre Moins que zéro (1986) et Les lois de l'attraction (1988) refuse American Psycbo, qui paraîtra ainsi chez Salvy avant d'être repris chez Seuil. 
des effets de réel. Le personnage-narrateur, Patrick Bateman, cumule les signes de l'idéal américain: 26 ans, analyse financier à Wall Street, corps soigneusement sculpté par les séances au club de gym, cheveux plaqués en arrière et lunettes Oliver Peoples à verres neutres et monture de séquoia, costume croisé Cerruti 1881 en gabardine de laine, Rolex au poignet, attaché case Tumi; on le croit souvent mannequin ou vedette de cinéma. Solidement inscrit dans sa réalité new-yorkaise - il habite le même immeuble chic que Tom Cruise - il n'a de soucis apparents que de s'entourer des objets qu'il faut posséder, de s'afficher dans les endroits qui comptent, d'être présenté à Donald Trump et, surtout, de soigner son allure:

Après avoir enfilé un short brodé Ralph Lauren, un sweater Fair Isle et des chaussons Enrico Hidolin de soie imprimée petits pois, j'attache un sachet de plastique empli de glace autour de mon visage et attaque les exercices d'assouplissement matinal. Après quoi, debout devant le lavabo Washmobile, résine et acier - assorti du porte-savon, du porte-gobelet et des barres d'acier pour poser les serviettes - que j'ai acheté chez Hastings Tile, en attendant que soit terminé le ponçage des lavabos de marbre que je fais venir de Finlande, j'observe mon reflet dans le miroir, le visage toujours entouré du sachet de glace. Je verse un peu de Plax anti-plaque dentaire dans un gobelet d'inox et le fait tourner dans ma bouche pendant une trentaine de secondes. Puis j'étale du Rembrandt sur une brosse en imitation écaille de tortue et entreprends de me brosser les dents (trop vaseux pour utilier le fil dentaire, ainsi qu'il conviendrait, mais peut-être l'ai-je fait hier soir, avant de me coucher?), et me rince la bouche avec de la Listerine. (p. 39)

La minutie descriptive sature tous les plans de la réalité: les restaurants et les boîtes à la mode (le Vanities, le Dorsia, le Club Chernoble, le Deck Chair, l'Ursula's, le Contra, le Beats, le World's End), les innombrables courses en taxi (du Bacardia au Tunnel, du Harry's au Nell's, à Upper West Side chez Evelyn, retour vers SoHo, escapade à Lower East Side), les conversations interminables sur les mérites comparés des diverses marques d'eau embouteillée (Sparcal, Perrier, San Pellegrino, Crodo, Poland Spring), l'étiquette vestimentaire (la règle en matière de pochette s'applique-t-elle aussi aux vestes de soirée? Existe-t-il une quelconque différence entre les chaussures de bateau et les TopSiders? Les cols ronds sont-ils trop habillés ou trop décontractés?), les systèmes électroniques sophistiqués (un récepteur 
audio, le Pioneer VSX-9300S comprenant le processeur Dolby Prologic Surround Sound intégré à programmation digitale, et une télécommande multi-fonctions à infra-rouges qui peut gérer jusqu'à 154 fonctions programmées sur tout autre appareil de la marque, et développant 125 watts sur le haut-parleur frontal, et 30 watts à l'arrière) de même que les adjuvants essentiels de toute cette panoplie bon chic bon genre, Valium, Xanax, Halcion, Nuprin, Extasy, Sominex, Dalmane, etc. La vie douillette, dorée, d'un "yuppie" à $30000 \$$ par mois, à peine un peu nerveux, brandissant à tout propos son AmEx platine qu'il utilise pour snifer ses lignes de coke et régler ses additions faramineuses. En arrière-plan, comme un décor fortement constrasté, la pauvreté affichée, les clochards qu'on houspille, la saleté et la laideur.

Peu à peu, la tension entre ce raffinement extrême et la plus sauvage barbarie s'instaure. De fait, la nuit, Pat Bateman se transforme en tueur fou, armé de machette, de perceuse électrique, de pistolet à clous et il décapite, poignarde, tronçonne ses victimes. Le même regard uni, qui vient de détailler le spectacle du cadavre énucléé et écorché, décrit de façon tout aussi neutre la scène d'anthropophagie:

Je passe les quinze minutes suivantes dans un état de semi-conscience, tirant sur un long morceau d'intestin bleuâtre encore solidaire du corps, et me le fourrant dans la bouche, jusqu'à l'étouffement. Il est humide contre mon palais, et rempli d'une espèce de pâte qui ne sent pas bon. Après une heure d'efforts, je parviens à détacher la moëlle épinière, que je décide d'expédier par Federal Express, sans la nettoyer, enveloppée dans des mouchoirs en papier, à Leona Helmsley, ceci sous un faux nom. Voulant boire le sang de cette fille comme si c'était du champagne, je plonge mon visage, profondément, dans ce qui reste de son estomac, et me mets à lapper, m'éraflant la joue contre une côte brisée. L'immense nouveau récepteur de télévision est allumé dans une des pièces, et l'on entend brailler le Patty Winters Sbow de ce matin, dont le thème était: Les Produits Laitiers Humains, puis un jeu télévisé, Wheel of Fortune, et les applaudissements du public semblent exactement les mêmes, à chaque fois que l'on retourne une nouvelle lettre. Je desserre ma cravate d'une main ensanglantée, prenant une profonde inspiration. Voilà ma réalité. En dehors de cela, tout m'apparaît comme un film que j'aurais vu autrefois. (p. 443)

Sauf à apprécier l'ironie froide qui préside à l'intégration des séries télévisées américaines, la référence se fait insoutenable. $\mathrm{La}$ 
caution littéraire manque pour permettre à la stratégie lectorale de surmonter, toute bonne conscience acquise, la répugnance du spectacle. Et c'est précisément dans ce jeu exacerbé de la description que réside l'interrogation fondamentale d'American psycho. Où est l'insupportable? Dans la violence même ou dans le récit qui la banalise?

\section{Fragmentation : premier et second degré}

On pourrait sans doute établir un parallèle convaincant entre le jeu de massacre nocturne de Bateman et la violence de la culture américaine, axée sur le culte du corps. L'exaltation du corps jouissif — sain, musclé et bronzé - est constamment représentée dans le récit par les séances répétées au club de gym, le raffinement alimentaire, les jeux de séduction, la préoccupation vestimentaire. Le souci maniaque de l'étiquette (que doit-on manger, boire, porter pour être intégré?), qui signale la subordination aux codes dominants, s'inverse en chiasme lors des activités inavouables de Bateman autant par le raffinement barbare des tortures infligées que par l'incongruité des mains manucurées de Bateman fouillant la chair sanglante de ses victimes ${ }^{2}$. L'exploitation récurrente de tels contrastes souligne, de façon oblique, le fascisme d'une certaine culture américaine fondée sur le mépris de la différence ${ }^{3}$.

Le parallèle s'affine encore par l'analogie qui peut se lire entre le dépecage systématique des corps et l'univers atomisé où s'exerce toute cette violence gratuite. Démembré, décapité, énucléé, violé, dissous dans la chaux vive à Hell's Kitchen, le corps massacré n'est pas sans évoquer l'identité culturelle morcelée du sujet contemporain, métaphoriquement écartelé au gré d'une civi-

2 À ce titre, l'épisode à propos du tableau de David Onica (50 000 \$) accroché la tête en bas est révélateur: Bateman tuera Bethany lorsqu'elle lui fera remarquer que la toile est à l'envers (p. 315). Auparavant, un commentaire de Bateman aura souligné le caractère emblématique du dit tableau : "Eh bien, je pense que son travail ... il y a là une sorte de ... de fausse légèreté voulue, magnifiquement équilibrée. Je m'interromps, essayant de retrouver la formule que j'ai lue dans un article de New York. "Une superficialité consciente..." " (p. 132)

3 Le récit accuse encore cette dimension par la convocation constante du mot nazi et les haines du personnage à l'endroit de ceux qu'il nomme youpins, nègres, macaronis, gouines, tantouzes, pédés. Sans oublier les clochards mendiants, qui l'exaspèrent et auxquels il conseille de se trouver un emploi, avant de les poignarder. 
lisation de l'instantané, qui juxtapose, cumule et recycle à vitesse folle des images, des sons, des bribes de textes, des affiches. L'activité diurne de Bateman, à la façon d'un vidéo-clip, est un kaléidoscope d'impressions colorées, une série de séquences hétérogènes où tout cependant apparaît toujours fragmentaire, déjà découpé, déjà filtré: le téléviseur, les baies vitrées qui surplombent la cité, la vitre du taxi, les lunettes, à verres noirs ou neutres, découpent le réel et forment écran.

De la même manière, la médiatisation de la culture, omniprésente, s'insère constamment dans la trame narrative par le biais de l'affiche des Misérables, sur laquelle on bute sans cesse et qu'on retrouve partout: placardée sur les bus, roulée dans la main d'un top model, sur la banquette arrière d'un taxi, souillée d'urine dans une ruelle, barbouillée du mot gouine en travers du visage d'Éponine. Bateman en volera même l'enregistrement original $\mathrm{CD}$ à son collègue qu'il vient d'assassiner à la hache, de même qu'il l'écoutera en compagnie des filles qu'il tronçonnera à la scie sauteuse.

"Je déteste la musique liven, répétera Bateman, son walkman vissé aux oreilles. La trame sonore, constamment décrite et commentée, fait défiler les stars du rock contemporain: Bon Jovi, Talking Heads, Guns n' Roses, les Tokens, Whitney Houston, Huey Lewis and the News (et tous leurs titres) se succèdent et donnent parfois lieu à des pages de commentaires évaluatifs qui ressemblent étrangement à des rubriques discographiques. L'amalgame de la réalité et de la fiction culmine encore dans la scène où Bono de U2 distingue Bateman dans la foule venue assister à leur concert (p. 192). "Je suis rock'n roll ", dira Bateman, citant son frère.

Dans cette civilisation de la citation, on répète interminablement ce qu'on a vu à la télé, ce qu'on a lu dans les quotidiens : Post, USA Today, New York, Times, The Nation, Imposture sont constamment sollicités. De même, on feuillette distraitement Sports Illustrated, Playboy, Vanity Fair, Gentlemen Quaterly, Esquire, Lesbian Vibrator Bitches, Cunt on Cunt, Women's Wair, Financial Times, le guide Zagat et les publicités de Saks. L'éclectisme de l'imprimé, substitué à la référence littéraire ${ }^{4}$, récupère

4 Deux titres seulement d'ouvrages littéraires sont récupérés par la narration: Docteur Jivago de Boris Pasternak et L'adieu aux armes d'Ernest Hemingway (p. 361). 
aussi les graffiti et les enseignes: le roman s'ouvre sur les mots "ABANDONNE TOUT ESPOIR, TOI QUI PÉNÈTRES ICI" barbouillés en lettres de sang au flanc de la Chemical Bank, et se ferme sur le panneau "SANS ISSUE", en lettres rouges. Tout au long du texte, des clochards brandissent des pancartes où on peut lire: "J'AI FAIM. JE SUIS SANS ABRI... AIDEZ-MOI SVP DIEU VOUS BÉNISSE.

Les émissions télé préférées, le Late Night with David Letterman, Murphy Brown ou le Club du Télé-Achat - que l'on regarde en faisant l'amour - sont passées et repassées en différé par le recours au magnétoscope, lequel devient par ailleurs le support de l'obsession pornographique: American psycho est un répertoire des films "pour adultes seulement", monté au gré des visites quotidiennes au club vidéo de Bateman. Mais c'est sans conteste l'évocation ad nauseam du Patty Winters Show, émission visionnée religieusement chaque matin et qui alimente toutes les conversations, qui traduit le mieux la charge ironique du texte ${ }^{5}$.

La mode, avec le design et la décoration, viendra cristalliser la fonction parodique, par l'insistance du texte à en faire le savoir le plus prisé, le summum du raffinement et de la culture. Le vêtement griffé, comme le service à thé Hermès, désigne le rapport au monde et devient le signe par excellence de la distinction, en même temps que celui de l'amalgame, le signe d'appartenance à la caste. Le texte d'American psycho est littéralement truffé de descriptions vestimentaires:

Nous sommes tous trois, David Van Patten, Craig McDermott et moi-même, installés dans la salle à manger du Yacht Club, en train de déjeuner. Van Patten porte un costume Krizia écossais, en crêpe de laine, une chemise Brooks Brothers, une cravate Adirondack et des chaussures Cole-Haan. McDermott porte un blazer en lambswool et cashmere, un pantalon en flanelle de laine peignée, Ralph Lauren, chemise et cravate également Ralph Lauren, et des chaussures Brooks Brothers. Je porte un costume de laine à motif écossais carreaux de fenêtres, une chemise de coton Luciano Barbera, une cravate Luciano

5 Les titres du Patty Winters Show évoquent avec éloquence les préoccupations éclatées d'une société qui se complaît à les commercialiser : Les Survivants des Camps de Concentration, Les Animaux qui Parlent, Les meilleurs Restaurants du Proche-Orient, Un Nouveau Sport: le Lancer de Nains, Les Descendants des Membres du Donner Party, Les Tueurs d'enfants, Parfums, Rouge à Lèvres et Maquillage, Rambo Existe, Je l'Ai Rencontré, Les Nazis, etc. (sic). 
Barbera, des chaussures Cole-Haan et des lunettes Bausch \& Lomb à verres neutres. [...] Luis Carruthers est assis à cinq tables de la nôtre, habillé comme s'il avait dû essuyer une espèce d'attaque de grenouilles au réveil - il porte un costume indéterminable, d'un créateur français indéterminé; et si je ne me trompe, le chapeau melon posé à terre, sous sa chaise, lui appartient aussi - c'est signé * Luis ", aucun doute. (p. 204-205)

La sémiologie de la mode est ici portée à son paroxysme et devient à la fois un véritable exercice de déchiffrement jumelé à un art consommé de la surenchère. Bateman est une machine à décoder le "look", à apprécier les combinaisons vestimentaires hybrides, à reconnaître les citations stylistiques. Parangon d'élégance, il tranche net et identifie au premier coup d'œil l'accessoire qui démarque le plouc de l'esthète: tout est dans le montage, le métissage savant. On pourrait aisément lier l'art vestimentaire de Bateman et l'écriture d'American psycho: dans les deux cas il s'agirait, comme le dit Scarpetta, "de répondre au risque de mauvais goût par un surcroît de mauvais goût, jusqu'au moment où cela se renverse en une élégance supérieure, désinvolte, au second degré " 6 .

\section{Le paradoxe de l'artifice}

Les descriptions innombrables ne tueront pas le récit. Au contraire, une telle outrance référentielle le met en évidence, signalant l'artificialité de cette société par l'artificialité formelle. D'entrée de jeu, le regard se désigne comme regard: "Comme dans un film, un autre bus entre dans le champ" (p. 10); "changement de plan. Retour à la cuisine" (p. 19); "Price attend une réaction. En vain. Soudain, ils sont dans l'Upper West Side" (p. 13). Le regard acéré découpe le réel en plans successifs, sans fondu enchaîné, marquant les ruptures.

En contrepoint, la fragmentation formelle accentue le portrait. Une soixantaine de scènes, aux titres prosaïques, atomisent le récit: Mardi, Nettoyage à sec, Canicide, Au magasin de vidéo/Chez D'Agostino, Dîner avec ma secrétaire, Où l'on tente de faire cuire une fille et de la manger, Shopping, Genesis, L'été, Au Nell's, Soins du visage, etc. Cependant, du fait qu'elles ne désignent pas des chapitres mais marquent des changements d'espace-temps, ces

6 Guy Scarpetta, L'artifice, Paris, Grasset, 1988, p. 124. 
multiples ruptures accentuent le caractère aléatoire de la narration: même si le récit peut sembler évoquer un journal tenu par le psychopathe, nulle motivation thématique ou chronologique n'informe le propos. Le temps est un continuum vide, ponctué de conversations futiles, de lignes de coke qu'on snife dans les toilettes des bars à la mode, sur fond démentiel de musique rock. L'artifice se signale encore par la répétition de certains titres, qui, tout comme l'acharnement descriptif, vient miner la visée représentative, en soulignant la notion de séries. Sémir Badir, dans un article intitulé "Vers la postmodernité", interroge la vanité du procédé:

les descriptions (de lieux publics, de costumes), qui ont la qualité truculente d'un Balzac ou d'un Huysmans, sont à ce point répétées, systématisées, comme une sorte de mécanique implacable, indifférente à toute utilité narrative, qu'elles en arrivent à s'annihiler et à rendre vaine du même coup toute entreprise de représentation. ${ }^{7}$

À ces considérations, il faudrait sans doute ajouter l'indétermination qui caractérise American psycbo et faire valoir qu'elle contamine à la fois l'identité des personnages, le tissu diégétique et le sens même du propos. Les personnages, clones interchangeables, se confondent les uns les autres, ne se reconnaissent pas mutuellement. Même l'aveu est refusé: en un moment de démence, $\mathrm{Ba}$ teman confie à un répondeur téléphonique le récit détaillé des meurtres commis; lorsqu'il rencontrera son confident, celui-ci, le confondant avec un autre, lui déclare qu'il a trouvé sa plaisanterie absolument suave, et lui démontre l'impossibilité de l'affaire puisqu'il a déjeuné avec une des prétendues victimes et que, de toute façon, Bateman est un tel peigne-cul, un tel cafard, une telle mijaurée, et impuissant par surcroît, qu'il ne pourrait jamais être l'auteur de tous ces méfaits. Bateman a-t-il vraiment tué, ou n'avons-nous eu droit qu'à des citations de "snuff movies"? Ne fait-il que nous raconter les tortures qu'il passe et repasse sur son magnétoscope? La convention romanesque, portée par une surréférentialisation permanente, est déboutée et le personnage-

7 Sémir Badir, "Vers la postmodernité ", Écritures, no 5, automne 1993, p. 17. Badir se fait le relais de la réception européenne, plus encline à apprécier la postmodernité du texte. Dans cette perspective, Bret Easton Ellis se rapproche d'un groupe d'écrivains américains (Jay McInerney, Ann Beattie, Bobbie Ann Mason, David Leavitt, Jill Eisenstadt, Tana Janowitz) rassemblés sous l'appellation d'xécrivains du désenchantement * dans la tradition de Fitzgerald. Ailleurs, on les nommera les "nouveaux minimalistes ". 
narrateur acquiert toute sa dimension parodique. La référence à la littérature mineure apparaît dans l'hybridation du personnage Bateman $^{8}$, qui inverse radicalement le Batman de Gottam City et sollicite le Dracula de Bram Stoker:

puis, mon parapluie ouvert, je me mets à courir dans Broadway, dans un sens, puis dans l'autre, vagissant comme une âme en peine, mon pardessus ouvert, voletant derrière moi comme une espèce de cape. (p. 217)

L'enjeu du récit est déporté et un second degré se signale comme la dénonciation de la déréalisation de la société contemporaine. $\mathrm{La}$ "salade de saucisses de mouton au homard et les haricots blancs arrosés de citron vert et de vinaigre de foie gras" (p. 382), que le jeu du réel nous faisait distraitement admettre, retrouve une fois reconnue la dimension ironique - son aspect artificiel de même qu'elle signale le projet esthétique en termes d'hybridation, de métissage d'éléments disparates, inconciliables.

Étrangement, toutefois, et de la même manière que Bateman se présente au party d'Halloween de son bureau déguisé en tueur maniaque (avec même une pancarte accrochée dans le dos sur laquelle on lisait MANIAQUE et portant en boutonnière une phalange qu'il avait fait bouillir pour bien nettoyer l'os, 424), affirmant qu'il a tué et sans être cru, l'ironie affichée demeure ambiguë. Comme si l'excès de réel, en prétendant déréaliser, avait raté sa cible et ne permettait plus de distinguer nettement, même dans l'ordre du récit, le vrai du faux. Là réside précisément le paradoxe de l'artifice, tel que le décrit Scarpetta:

Le paradoxe est celui-ci: lorsque le second degré se généralise (lorsque tout peut fonctionner comme une citation), il cesse du même coup d'exister; par une sorte de ruse dialectique, tout finit par être perçu sur le même plan (donc, au premier degré). ${ }^{9}$

La frontière, dans American psycbo, demeure perméable. Montés par le texte, la joute perpétuelle entre le premier degré et l'ironie, le va-et-vient constant entre le fanstasme et la réalité, la tension permanente entre la neutralité du regard et l'outrance du narré, désarçonnent et amorcent les effets de réception aberrants.

8 On pourrait épiloguer sur le nom même du personnage et faire ressortir la dimension parodique du patronyme: bate, en anglais, signifie réduire, diminuer, retrancher.

9 Guy Scarpetta, op. cit., p. 138. 
104

L'absence de sanction diégétique et l'absence de code littéraire affiché viennent conforter l'enjeu paradoxal du texte en termes d'une déréalisation qui se nourrit de l'outrance du réel.

\section{En toute impunité}

La modernité littéraire nous avait habitués à chercher les signes d'une inscription dans le champ littéraire. Ici, rien de tel: pas d'auteur qui se désigne en train d'écrire, pas de référence à lacte scriptural (à peine verra-t-on Bateman se filmer lui-même en train d'assassiner et d'éventrer), pas d'indice critique. À l'inverse de Bateman, qui affiche ostensiblement son appartenance, son fric, ses fringues, le texte ne se désigne pas "littérature" et s'apparente aux discours futiles des personnages :

La conversation suit sa pente naturelle - ni structure proprement dite, ni sujet précis, ni logique interne, ni sentiment; à part, bien sûr, ce sentiment de dissimulation et de complot inhérent à toute conversation. (p. 508)

La dissimulation et le complot, s'ils existent, résident dans cette écriture finement ciselée, tellement "objective" qu'elle se dissimule comme écriture. Paroxysme de la référentialisation et diabolisation non immédiatement littéraire peuvent être interprétés comme une neutralisation de l'écriture, qui se saborde elle-même. Là où la littérature du mal - Sade, Lautréamont, Artaud, Bataille - exploitait sexe et sang en une volonté avérée de transgression, American psycho ne joue pas de l'interdit: banalisée, ironisée, kitchéisée, la violence est sans cause, sans propos, sans motif, absolument et essentiellement gratuite. La loi littéraire n'est ni évoquée, ni mise en scène, pas plus interrogée que déboulonnée: entre la bande dessinée et la satire sociale, le texte donne à voir.

L'écriture froide et neutre de Ellis, mâtinée de Balzac et de Sade, est à l'image de son personnage-narrateur. Comme lui, elle dissimule ses pulsions meurtrières sous un masque d'élégance stylisée; comme lui, elle met à mal l'intégrité de la norme narrative; comme lui, elle est "la caricature du sujet postmoderne qui ne croit plus aux frontières entre le vrai et le faux, entre l'originalité et la duplication" ${ }^{10}$; comme lui, elle pousse le mauvais goût

10 L'expression est de Karen Gould, ‘Copies conformes: la réécriture québécoise d'un polar américain ", Études françaises, vol. XXIX, nº 1, 1993, p. 31. 
jusqu'à la limite de la désinvolture, pastichant allègrement le roman noir, la vidéo porno, le Patty Winters Show et le catalogue Armani. Et, comme lui, elle restera impunie.

Mais pourquoi nous raconte-t-il toutes ces conneries? 